\title{
Intubation practice patterns in Tuscan emergency departments
}

\author{
Leon D. Sanchez • Paolo Di Martino • \\ Matthew Babineau • Michele Lanigra • Kevin M. Ban
}

Received: 23 January 2008 / Accepted: 5 March 2008/Published online: 29 May 2008

(C) Springer-Verlag London Ltd 2008

\begin{abstract}
Introduction Intubation is one of the most important lifesaving procedures performed by emergency physicians (EPs). There is variation in practice when different countries are compared.

Methods A written questionnaire on intubation practices was administered to a group of Italian doctors practicing in Tuscany during the examination period of a year-long course in emergency medicine.

Results The survey was administered to 153 participants. Of these, $143(93.4 \%)$ returned a complete survey. In the sub-group of physicians who work in the emergency department (ED), $73.6 \%$ report intubating patients. Of those that intubate patients, $92.3 \%$ use some sort of sedation, and $49.3 \%$ use paralytics. While direct visualization of the cords for intubation and auscultation of breath sounds after intubation are almost universal $(97 \%$ and $100 \%$, respectively), only $11.9 \%$ use colorimetric CO2 detectors for confirmation of intubation. After intubation $58.2 \%$ commonly place a nasogastric tube and 50.7\% obtain a post intubation chest radiograph.
\end{abstract}

Sources of support: none

L. D. Sanchez $(\bowtie) \cdot$ M. Babineau $\cdot$ K. M. Ban

Department of Emergency Medicine,

Beth Israel Deaconess Medical Center,

One Deaconess Road WCC-2,

Boston, MA 02215, USA

e-mail: Leon_sanchez@bidmc.harvard.edu

P. Di Martino

Tuscan Emergency Medicine Initiative,

Tuscany, Italy

M. Lanigra

U. O. Emergenza ed Accettazione Ospedale

Misericordia e Dolce USL4 Prato,

Prato, Italy
Conclusions Practice patterns in the USA and Tuscany are different. RSI and post-intubation radiographs are the standard of care in EDs in the USA. This is not the case in Tuscany.

Keywords Airway Intubation · Emergency medicine

\section{Introduction}

Emergency medicine (EM) is an established specialty in the USA. Intubation is one of the most important life-saving procedures performed by emergency physicians (EPs) and is considered a defining skill of the specialty [1]. After years of standardized residency training in EM, intubation practice in the USA has become uniform with rapid sequence intubation (RSI) representing the widely accepted standard of care. In other countries where EM is at an earlier stage in its professional development, larger practice variations in airway management may exist.

The Tuscan Emergency Medicine Initiative is a comprehensive program initiated in 2003 to create a sustainable emergency medicine (EM) training and qualification process in Tuscany, Italy. The program is a collaboration among the Tuscan Ministry of Health, the Universities of Florence, Pisa and Siena, the Harvard Medical Faculty Physicians at Beth Israel Deaconess Medical Center and Harvard Medical International. The program has the goal of training several hundred practicing EPs. At the inception of the program there was no official recognition of EM as a primary training specialty.

The qualification course for practicing EPs is a 12-month part-time course. Physicians need a minimum of 3 years of work in the emergency department (ED) or 5 years in the prehospital system to be eligible to participate in this course. Successful course completion leads to physician 
recognition by the regional government. The program includes 8 weeks of clinical rotations, 5 full-day workshops, and 48 1-h lectures based on the American and European core contents of EM [2, 3]. One of these workshops as well as some lectures were dedicated to airway management. At the end of the course, written and oral exams were administered to all participants who successfully completed the training. At the end of the written exam a survey regarding intubation practices was answered by all the candidates.

\section{Methods}

A written questionnaire was administered at the end of the written exam for the qualification course. A total of 153 people received the survey. These were all doctors that worked either in the ambulance system or the emergency department. Questions were asked regarding type of work environment (ambulance or ED), whether the physician performed intubations, use of medications and methods to confirm endotracheal tube placement (Table 1). Data were entered and analyzed with a Microsoft Excel 2003 (Redmond, WA) database. The IRB waived consent for the study.

\section{Results}

The survey was administered to 153 participants. Of these $143(93.4 \%)$ completed the survey. Of the survey participants $52(36.4 \%)$ were doctors in the ambulance system and $91(63.6 \%)$ were doctors that worked in the ED. All doctors that work in the ambulance system need to intubate as part of their job. Their primary method of confirmation of placement is direct visualization and auscultation. Since these are prehospital intubations they are normally intubations done in arrest situations or for patients in extremus. Because of the setting limited resources are available, and few if any medications are utilized. Many of the peri- and post-intubation questions in the survey are not relevant to this group. The ambulance physicians were not included in the rest of the analysis since most of the questions were not relevant to their scope of practice.

Complete information about the results can be seen in Table 1.

A total of $73.6 \%$ of ED-based physicians report intubating patients. Of those that intubate, $92.3 \%$ use sedation and $49.3 \%$ use paralytics. No physician was using paralytics in the absence of sedation. While direct visualization of the cords during intubation and auscultation of breath sounds
Table 1 Questionnaire information

\begin{tabular}{|c|c|c|}
\hline & \multicolumn{2}{|l|}{ Summary of data } \\
\hline & $\mathrm{N}$ & $\%$ \\
\hline Surveys administered & 153 & \\
\hline Surveys completed & 143 & $93.4 \%$ \\
\hline MDs working in ED & 91 & $63.6 \%$ \\
\hline MDs from ambulance service & 52 & $36.3 \%$ \\
\hline ED doctors that intubate in their ED & 67 (out of 91) & $73.6 \%$ \\
\hline \multicolumn{3}{|l|}{ Of those that intubate: } \\
\hline Use of paralytics & 33 & $49.3 \%$ \\
\hline Sedation & 132 & $92.3 \%$ \\
\hline Midazolam & 45 & $59.7 \%$ \\
\hline Propofol & 31 & $46.3 \%$ \\
\hline Fentanyl & 14 & $20.9 \%$ \\
\hline \multicolumn{3}{|l|}{ Methods of confirmation } \\
\hline Direct visualization & 65 & $97 \%$ \\
\hline Auscultation of breath sounds & 67 & $100 \%$ \\
\hline $\mathrm{CO} 2$ detector & 8 & $11.9 \%$ \\
\hline \multicolumn{3}{|l|}{ Post intubation } \\
\hline Nasogastric tube placement & 39 & $58.2 \%$ \\
\hline Chest radiograph & 34 & $50.7 \%$ \\
\hline \multicolumn{3}{|c|}{ Questionnaire administered (translated from the Italian) } \\
\hline Place of work: & \multicolumn{2}{|c|}{ Ambulance or hospital } \\
\hline Do you intubate & \multicolumn{2}{|c|}{ Yes/no } \\
\hline Do you use sedation & \multicolumn{2}{|l|}{ Yes/no } \\
\hline If yes, what medications & & \\
\hline Do you use paralytics & \multicolumn{2}{|l|}{ Yes/no } \\
\hline If yes, which paralytics & & \\
\hline \multicolumn{3}{|c|}{ For ET tube placement confirmation do you: } \\
\hline Auscultate & Yes/no & \\
\hline Directly visualize & The cords yes/no & \\
\hline Use $\mathrm{CO} 2$ detector & Yes/no & \\
\hline $\begin{array}{l}\text { Routinely place an NGT post } \\
\text { intubation }\end{array}$ & & Yes/no \\
\hline $\begin{array}{l}\text { Routinely obtain a post intubation } \\
\text { radiograph }\end{array}$ & & Yes/no \\
\hline
\end{tabular}

after intubation are the most common methods used to confirm correct endotracheal tube (ETT) placement $(97 \%$ and $100 \%$, respectively), only $11.9 \%$ use colorimetric $\mathrm{CO} 2$ detectors for confirmation of tracheal tube placement.

After intubation, $58.2 \%$ routinely place a nasogastric tube, and $50.7 \%$ obtain a post-intubation chest radiograph.

\section{Limitations}

This was a survey study and therefore relies on the accuracy of the answers of the participants. It was limited to one region of Italy so it may not reflect intubation practices in the whole country. 


\section{Discussion}

While airway management is part of what defines EPs in the USA, this may not be the case throughout the world. Emergency medicine is not a recognized specialty in Italy. Because of this there is a wide variation in the physicians that practice in EDs. Although the majority of doctors that work in the Tuscan EDs intubate patients, there are several differences in practice between EPs in Tuscany and in the US. In many EDs intubations are primarily or exclusively done by anesthesia. Even in EDs where EPs intubate, they may only be doing so in arrest or peri-arrest situations.

The use of paralytics for intubation together with sedation was used at least sometimes by only $49.3 \%$ of Tuscan EPs. This is in contrast to uniform training in RSI during residency and a $78 \%$ to $84 \%$ rate of RSI use in the US $[4,5]$. We can only speculate as to the reason for this, but training, level of comfort with the performance of the procedure as well as availability of medications may play a role.

The use of clinical methods to confirm ETT placement, both visualization of the cords and auscultation of breath sounds, is almost universal. Secondary methods of ETT confirmation are less widespread. While colorimetric CO2 detectors have been recommended for quick confirmation of endotracheal intubation, their use is not widespread in Tuscany [6]. This may be due to practice patterns or lack of availability of the device. We did not ask whether the device was available in the practitioner's ED, so cannot differentiate between these two possibilities.

Post intubation chest radiographs are routinely obtained in the US after emergency intubation. Several studies recommend its use [7-9]. This is not the case in Tuscany where only $50.7 \%$ of subjects say they routinely obtain a post-intubation chest radiograph. Many of the EPs that do not routinely obtain a post-intubation radiograph feel that clinical methods for confirmation of tube position are adequate. They point to anesthesiologists in the operating room and the fact that radiographs are not the standard of care post-intubation in this group. Whether these two groups are comparable is debatable.

In retrospect, questions regarding the availability of specific medications and colorimetric $\mathrm{CO} 2$ detectors as well as barriers to obtaining chest X-rays would have given us more information that may have helped deliniate some of the reasons for differences in practice.

\section{Conclusions}

In summary, practice patterns in the US and Tuscany are different. Management of the airway by EPs, RSI and postintubation radiographs are the standard of care in EDs in the US. This is not the case in Tuscany. We would expect the rate of RSI to increase as EPs in Tuscany receive specific training and become more comfortable with the procedure. Evaluation of the utility of post-intubation radiographs warrants prospective study in Tuscany. Validation of the utility of the practice in the US may also be indicated.

\section{References}

1. Rosen P, Ban KM, Pini R (2006) Airway management: the sine qua non of emergency medicine. Intern Emerg Med 1(2):137-138

2. Hockberger RS, Binder LS, Graber MA, on behalf of the American College of Emergency Physicians Core Content Task force II (2001) The model of the clinical practice of emergency medicine. Ann Emerg Med 37(6):745-770

3. Petrino R, Bodiwala G, Meulemans A, Plunkett P, Williams D, on behalf of the European Society for Emergency Medicine (EuSEM) (2002) EuSEM core curriculum for emergency medicine. Eur J Emerg Med 9(4):308-314

4. Sagarin MJ, Barton ED, Chng YM, Walls RM (2005) Airway management by US and Canadian emergency medicine residents: a multicenter analysis of more than 6,000 endotracheal intubation attempts. Ann Emerg Med 46(4):328-336

5. Sakles JC, Laurin EG, Rantapaa AA, Panacek EA (1998) Airway management in the emergency department: a one-year study of 610 tracheal intubations. Ann Emerg Med 31(3):398-400

6. Bozeman WP, Hexter D, Liang HK, Kelen GD (1996) Esophageal detector device versus detection of end tidal carbon dioxide level in emergency intubation. Ann Emerg Med 27(5):598-599

7. Brunel W, Coleman DL, Schwartz DE, Peper E, Cohen NH (1989) Assessment of routine chest roentgenograms and the physical examination to confirm endotracheal tube position. Chest 96 (5):1043-1045

8. Schwartz DE, Lieberman JA, Cohen NH (1994) Women are at greater risk than men for malpositioning of the endotracheal tube after emergent intubation. Crit Care Med 22(7):1127-1131

9. Levy FH, Bratton SL, Jardine DS (1994) Routine chest radiographs following repositioning of endotracheal tubes are necessary to assess correct position in pediatric patients. Chest 106(5):15081510 